\title{
Efecto que tiene el radio de la cabeza en la percepción y localización de sonidos
}

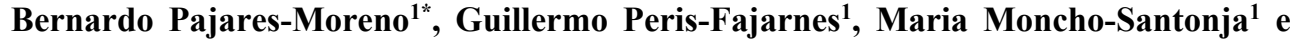 \\ Ismael Lengua ${ }^{1}$ \\ ${ }^{I}$ C.I.T.G. Universitat Politècnica de València, camino de Vera s/n, 46022 Valencia; \\ *E-mail address: berpamo@etsia.upv.es
}

\begin{abstract}
In the present article the methodology followed for the realization of the experimental is defined, which aims to evaluate how the head radio affects the perception and location of sounds. It has been tried to evaluate the perception of the sound on the part of the human being and to try to improve it by means of a series of experiments that have as reference the Relative Function to the Head (HRTF).
\end{abstract}

Keywords: Binaural; intertemporal; interaural; location; head radio.

\begin{abstract}
Resumen
En el presente artículo se define la metodología seguida para la realización del experimental, que pretende evaluar cómo afecta el de radio de la cabeza en la percepción y localización de los sonidos. Se ha pretendido evaluar la percepción del sonido por parte del ser humano e intentar mejorarla mediante una serie de experimentos que tienen como referencia la Función de Transferencia Relativa a la Cabeza (HRTF).
\end{abstract}

Palabras clave: Binaural; intertemporal; interaurales; localización; radio de la cabeza.

\section{Introducción}

Alrededor del $2 \%$ de la población europea sufre de deficiencia visual, por lo que la necesidad de un dispositivo de una ayuda para su autonomía e integración personal se vuelve un hecho fundamental. Existe una amplia gama de dispositivos de navegación disponibles en el mercado para ayudar a este tipo de usuarios. Los más comunes son: Electronic Travel Aid (ETA), Electronic Orientation Aid (EOA) y Position Locator Devices (PLD's). 
La Universidad Politécnica de Valencia (UPV) comenzó a colaborar en el proyecto europeo CASBLIP con el objetivo de desarrollar una herramienta tecnológica capaz de integrar socialmente a personas con problemas de visión. Como resultado de CASBLIP, el proyecto de Eye 20/21 fue creado por el Centro de Investigación en Tecnologías de Gráficos (CITG) de la UPV, coordinado por Guillermo Peris Fajarnés.

Eye 21 son gafas que se basan en un principio de la traducción, de visual a sonido, lo que permite al usuario ser capaz de reconocer lo que está delante solo a través del oído, sin la necesidad de ver.

Las gafas están equipadas con micro cámaras capaces de reunir la información visual de los objetos que nos rodean; esta información se traduce en sonidos relacionados con objetos, que a través de los auriculares llegan al receptor.

Para mejorar la funcionalidad del dispositivo, hasta la fecha, la idea es crear un "mapa de sonido 3D", para que nuestro cerebro pueda transformar los sonidos en imágenes que representen la realidad que nos rodea. El objetivo es obtener una percepción del volumen de un objeto, de su forma, su tamaño, su altura y, finalmente, su color. De esta manera, el espacio no se puede ver, pero se puede sentir.

\section{Desarrollo}

\subsection{Objetivo}

Con el objetivo de crear un mapa tridimensional de sonidos, el trabajo de investigación en el CITG (Centro de Investigación en tecnologías de gráficos de la UPV) propone como un primer paso, evaluar la percepción de las capacidades de sonido por parte de las personas, actuando sobre los parámetros fundamentales involucrados en su percepción.

\subsection{Introducción}

El ser humano es capaz de distinguir el origen de un sonido por medio de diferentes mecanismos. El fundamental se basa en la escucha binaural, es decir, con ambos oídos. A través de esta escucha, nuestro sistema perceptivo puede comparar las características de un sonido que llega a los oídos y obtener información sobre la posición de la fuente que lo generó. La localización de los sonidos de alta frecuencia se basa en el IID, una diferencia interna en intensidad. Mientras que la localización de los sonidos de baja frecuencia se basa en la diferencia de tiempo interaural (ITD). Teoría que se llama Duplex Theory. Se sabe a partir de la literatura que las partes anatómicas del hombre interfieren con las ondas sonoras 
emitidas por una fuente acústica debido a los fenómenos de reflexión y difracción. La forma de la oreja, la cabeza, el torso y los hombros afectan la recepción de una onda de sonido. En particular, la conformación de la cabeza induce una DIT. Se hace referencia a ITD cuando la intensidad del sonido percibido por un oído puede ser diferente de la percibida por el otro oído, ya que la cabeza se coloca como un obstáculo entre una oreja y otra. Por lo tanto, ITD se define como el retraso interaural, es decir, el retraso que la onda de sonido, una vez que llega al oído más cerca de la fuente, tarda en llegar al otro oído más alejado.

Asumiendo un esquema en el que la cabeza de un sujeto (superficie esférica) se coloca en el origen de un semicírculo cuyo radio representa la distancia entre el sujeto y la fuente del sonido, el valor de la ITD o la distancia de tiempo extra necesaria para una el rayo de sonido para llegar al oído más distante se mide en segundos y se describe mediante la fórmula de Woodworth:

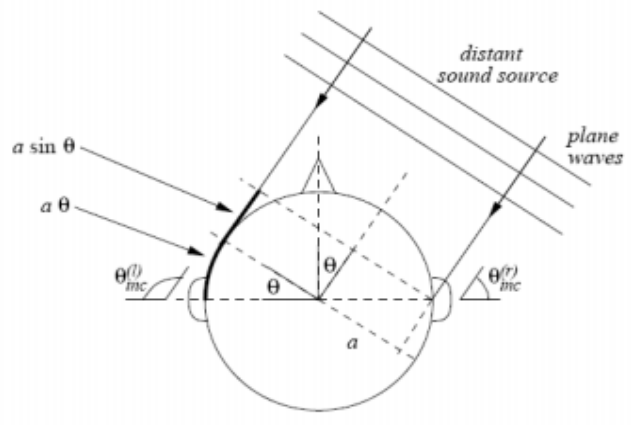

$\operatorname{ITD}=\frac{\mathrm{a}(\sin \theta+\theta)}{\mathrm{c}}$

$\mathrm{a}=$ radio de la cabeza;

$\mathrm{c}=$ velocidad del sonido en el aire;

$\theta=$ Ángulo acimutal (define la dirección de la onda de sonido en el plano horizontal en relación con el punto que coincide con la punta).

Fig. 1 Demostración formula ITD

El campo de visión es un área que representa la parte del mundo externo visible cuando un punto es fijo. Podemos distinguir tres regiones del campo visual: la visión periférica distante que incluye las áreas y los extremos del campo visual, la visión periférica promedio y la visión periférica cercana que incluye las áreas inmediatamente adyacentes al centro de la mirada. 


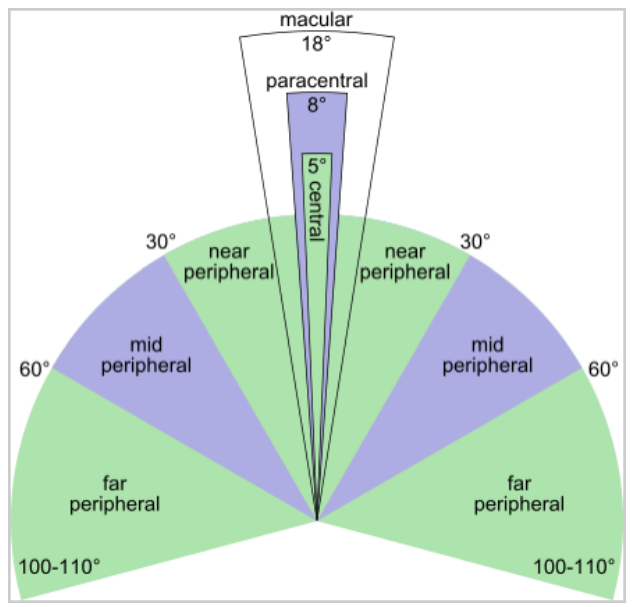

Fig. 2 Visión perimetral humana

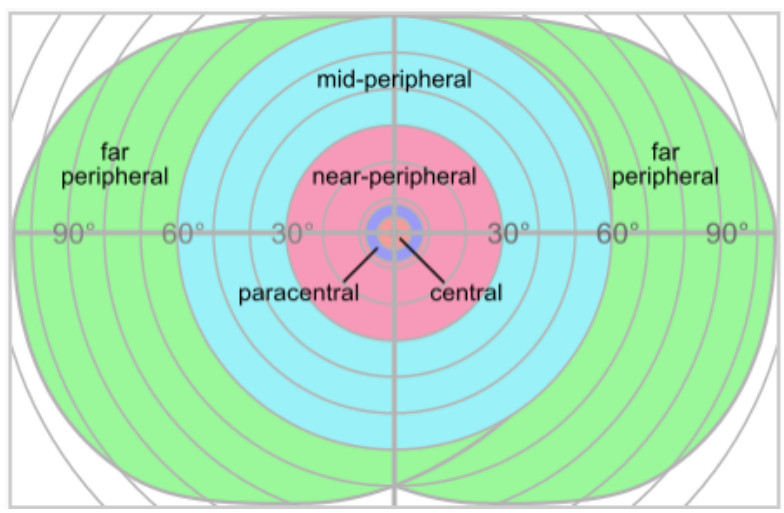

Fig. 3 Campo de visión humana

Imaginando que estamos en una región que se extiende hasta una visión periférica distante y suponiendo una fuente de sonido que puede moverse dentro de esta región, hemos hipotetizado un esquema en el cual se coloca la cabeza de un sujeto, supuestamente esférico, en el origen de un semicírculo cuyo radio representa la distancia entre el sujeto y la fuente del sonido. 
También se supuso que la fuente puede moverse a lo largo del semicírculo en los puntos A, B, C, D, E, F, G, H, I coincidiendo con diferentes valores de ángulo acimutal, como se muestra en la Figura 2.

Además, la altura de la fuente de sonido no cambia y se fija a un valor igual a la altura de la cabeza.

Finalmente, el origen del sistema de referencia se ha ubicado en el centro del semicírculo.

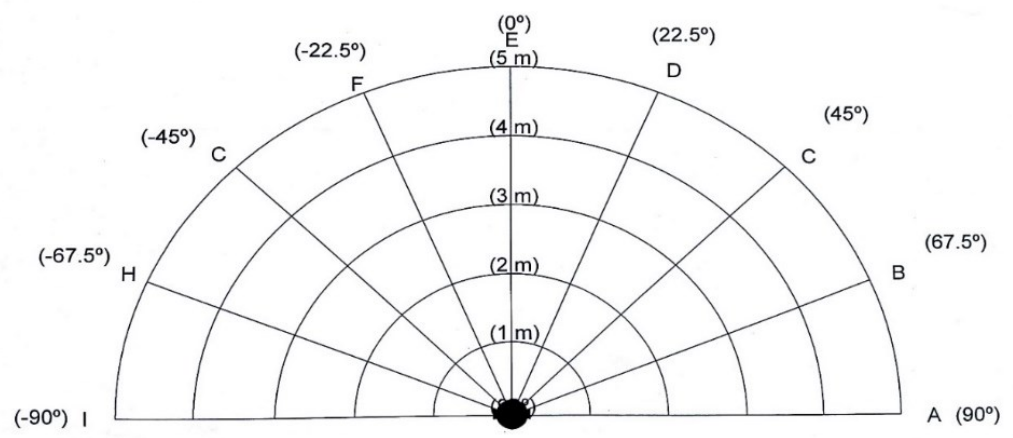

Fig. 4 Posición de la fuente de sonido

\subsection{Metodología}

En primer lugar, se descargaron dos tipos de sonido del sitio web "www.freesound.org", un sonido de metal y un sonido de cristal. El objetivo es manipular estos dos sonidos diferentes para reproducir la escucha binaural, prácticamente realizar DIT, a veces adaptando la frecuencia y la intensidad.

Por lo tanto, tanto el sonido metálico como el sonido de cristal se manejaron y procesaron utilizando el software Audacity y Matlab.

\subsubsection{Pruebas experimentales}

Para realizar las pruebas experimentales, se crearon algunas listas de reproducción que contienen las pistas de sonido con los sonidos procesados previamente.

Las pruebas experimentales se llevaron a cabo en una cámara anecoica, con un grupo de 9 voluntarios. La cámara anecoica es un entorno de laboratorio estructurado para reducir al máximo la reflexión de las señales en las paredes y simular campo abierto.

Las pruebas consisten en escuchar la lista de reproducción de sonidos, para verificar en función de los resultados obtenidos, cómo cambia la percepción promedio del sonido. Antes de poder realizar la prueba, fue necesario enviar a los voluntarios a una prueba audiométrica 
para descartar cualquier problema auditivo. La prueba audiométrica está disponible en el sitio web "www.beltonehearingtest.com" y la duración es de 4 minutos.

Los experimentos se llevaron a cabo usando auriculares bluetooth (JBL, auriculares inalámbricos T450BT que cubren todo el pabellón auditivo).

Los oyentes recibieron esquemas, de tal manera que, una vez sometidos a pruebas auditivas en una cámara anecoica, podían marcar gráficamente el punto donde escuchaban el sonido.

\section{Conclusiones}

La metodología de trabajo utilizada para la creación del mapa tridimensional de sonidos no ha dado el resultado esperado. Los usuarios obtenían datos dispares a la hora de localizar los sonidos, ubicándolos en el gráfico que se les facilitaba una vez que escuchaban cada sonido. En conclusión nos lleva a replantear la prueba, utilizando otro banco de sonidos que pueda ser más adecuado para posicionar espacialmente un sonido/elemento en el espacio. Por lo tanto, será necesario modificar su posicionamiento espacial del sonido e incidir en los parámetros experimentales necesarios para poder diseñar un nuevo método de trabajo con localizaciones más espaciadas, para calcular con precisión, cual es la resolución máxima, en distancia, que un oído humano es capaz de diferenciar al localizar un sonido en el espacio.

\section{Referencias}

Bronkhorst, A. W. (1995). Localization of real and virtual sound sources. The Journal of the Acoustical Society of America 98, 2542.

Brughera, A., Dunai, L., Hartmann W. M. (2013). Human interaural time difference thresholds for sine tones: The high-frequency limit. The Journal of the Acoustical Society of America 133, 2839.

Duda, R. O., Martens, W. L. (1998). Range dependence of the response of a spherical head model. The Journal of the Acoustical Society of America 104, 3048.

Emiliano Ricciardi, Daniela Bonino, Lorenzo Sani, Tomaso Vecchi, el al. (2009). Do We Really Need Vision? How Blind People "See" the Actions of Others. The Journal of Neuroscience 9719-24.

Gardner, W. G., Martin, K. D. (1995). HRTF measurements of a KEMAR. The Journal of the Acoustical Society of America 97, 3907.

Hafter, E. R., Dye, R. H. (1983). Detection of interaural differences of time in trains of high-frequency clicks as a function of interclick interval and number. The Journal of the Acoustical Society of America 73, 644. 
Kuhn, G. F. (1977). Model for the interaural time differences in the azimuthal plane. The Journal of the Acoustical Society of America 62, 157.

Lessard, N., Pare, M., Lepore, F., Lassonde, M. (1998). Early-blind human subjects localize sound sources better than sighted subjects. Nature, vol 395, 17 septiembre.

McFadden, D., Pasanen, E. G. (1976). Lateralization at high frequencies based on interaural time differences. The Journal of the Acoustical Society of America 59, 634.

Ohuchi, M., Iwaya, Y., Suzuki, Y., Munekata, T. (2006). Cognitive-map formation of blind persons in a virtual sound environment. Proceedings of the 12th International Conference on Auditory Display, London, UK June 20 - 23.

Peris, G., Dunai, L., Santiago, V., Dunai, I. (2010). CASBliP-a new cognitive object detection and orientation system for impaired people. 4th International Conference on Cognitive System ETH Zurich, Switzerland.

Saberi, K., Antonio, J. V. (2003). Precedence-effect thresholds for a population of untrained listeners as a function of stimulus intensity and interclick Interval. The Journal of the Acoustical Society of America 114, 420.

Wightman, F. L., Kistler, D. J. (1992). The dominant role of low-frequency interaural time differences in sound localization. The Journal of the Acoustical Society of America 91, 1648. 